\title{
Transcriptional Impact of E-cadherin Loss on Embryonic Stem Cells
}

\author{
Hani Alotaibi ${ }^{1,2, *}$ \\ ${ }^{1}$ Izmir Biomedicine and Genome Center, Izmir, Turkey; ${ }^{2}$ Izmir International Biomedicine and Genome Institute, Dokuz Eylül University, \\ Izmir, Turkey
}

Received: April 22, 2021; Revised: August 15, 2021; Accepted: August 24, 2021

\begin{abstract}
In embryonic stem cells, E-cadherin plays a crucial role in sustaining the pluripotent state; this is achieved by the simultaneous presence of active core pluripotency transcriptional network, proper cell-cell adhesion, and an undifferentiatedstate chromatin signature. In contrast, N-cadherin is linked to a more differentiated cell state but can support pluripotency if expressed as a knock-in allele from the E-cadherin locus. This study describes the N-cadherin ki/ki embryonic stem cells, which lack E-cadherin expression, to identify transcriptional changes distinct from the known changes observed in Ecadherin knockout embryonic stem cells. As a result, a remarkable similarity in the expression profiles of N-cadherin ki/ki and wild-type embryonic stem cells was observed. Further analysis of the slight differences revealed significant alterations in several biological processes such as chromatin organization and epithelial-mesenchymal transition. The findings presented here shed light on a new aspect of E-cadherin biology in embryonic stem cells and lay the foundations for comprehensive understanding of E-cadherin's functional relevance beyond the prominent role in maintaining pluripotency.
\end{abstract}

Keywords: E-cadherin, Epithelial to Mesenchymal Transition, EMT, Embryonic Stem Cells, Gene replacement.

\section{Introduction}

Embryonic stem (ES) cells can self-renew, maintain an undifferentiated state of pluripotency, and generate cells that can differentiate into the various cell types of the body (Niwa, 2007). The pluripotent state's maintenance requires the function of a core transcriptional network comprised of three main transcription factors, Nanog, Sox2, and Oct4 (Ying et al., 2008). The functions of these transcription factors keep the cells in an undifferentiated state distinguished by growth in compact colonies, an active pluripotency transcriptional network, and the presence of bivalent histone modifications on the promoters of several essential genes (Mikkelsen et al., 2007). The transcription factors Sox2 and Oct4, Klf4, and c-Myc induce the pluripotent state in mouse somatic cells by establishing ES-like cells called induced pluripotent stem cells (iPSCs) (Takahashi and Yamanaka, 2006). Nanog's activation is an essential step in establishing the pluripotent state (Silva et al., 2009). The reprogramming to the pluripotent state requires completing a well-orchestrated series of events, including changes in the chromatin landscape and DNA methylation states (Boyer et al., 2006, Maherali et al., 2007). These events coincide with the initiation of the mesenchymal to epithelial transition (MET), during which the somatic cells lose their fibroblast-specific mesenchymal morphology and gradually establish polarization and gain the ES cell-like appearance (Li et al., 2010). This MET event is a crucial step and depends on E- cadherin's (E-cad) activation, a calcium-dependent adhesion molecule. E-cad is essential for maintaining stemness and the pluripotent state since its loss can cause alterations in homophilic adhesion, causing a scattered cell growth of ES cells in culture (Larue et al., 1996, Soncin et al., 2009). Moreover, exogenous E-cad expression can substitute Oct4 in the mixture of reprogramming factors, which results in effective iPSC reprogramming (Redmer et al., 2011).

ES cell homeostasis fundamentally requires the E-cadmediated adhesion, an indispensable event for cell-cell communication and signaling (Chen et al., 2010). On the other hand, in mesenchymal cells, such as fibroblasts, cellcell adhesion is achieved by $\mathrm{N}$-cadherin ( $\mathrm{N}$-cad). The protein sequences of both cadherins show striking conservation; thus, they have a similar role in mediating homophilic adhesion and even interact with similar intracellular proteins such as beta-catenin. However, they are primarily present in a mutually exclusive fashion. These molecules also promote contrasting phenotypes; Ecad expression will result in a polarized epithelial form; in contrast, N-cad expression correlates with a depolarized motile state (Wheelock et al., 2008). Several studies addressed the functional redundancy of the two cadherins, their impact on critical developmental stages, and their impact on the pluripotent state in ES cells. One report investigated the impact of replacing E-cad by N-cad during trophectoderm formation and concluded that E-cad functions could not be restored by N-cad (Kan et al., 2007). Besides, the expression of $\mathrm{N}$-cad from the E-cad

\footnotetext{
* Corresponding author e-mail: hani.alotaibi@ibg.edu.tr

**Abbreviations: Embryonic stem (ES), induced pluripotent stem cells (iPSCs), E-cadherin (E-cad), N-cadherin (N-cad), knockout (ko), knock-in (ki), Gene Expression Omnibus (GEO).
} 
locus was sufficient to maintain the pluripotent state and was also suitable for reprogramming fibroblasts into iPSCs (Bedzhov et al., 2013). The maintenance of adhesion was enough for the preservation of pluripotency. Maintaining the pluripotent state by $\mathrm{N}$-cad in ES cells provided a unique opportunity to study the impact of E-cad loss on ES cells that retain the pluripotent state. In recent years, a great deal of research has been dedicated to studying ES cells and iPSCs, notably for their potential use in personalized medicine (Yamanaka, 2020). So, it is of interest to have a better understanding of stem cell biology in general, and a better understanding of the outcomes of potential gene expression signature changes would be of utmost importance for the design and planning of such therapies. The current study reveals the changes in ES cells' gene expression profiles expressing $\mathrm{N}$-cad instead of E-cad. This is addressed by performing in silico analyses and then comparing the expression profiles of E-cad knockout (ko) cells and N-cad knock-in (ki) cells to the wild-type ES cells.

\section{Materials and Methods}

\subsection{ES cell culture}

ES cells used in this work were described previously; in summary, $\mathrm{N}$-cad ki/ki, E-cad ko/ko, and wt ES cells were prepared from E2.5 embryos as described in (Bedzhov et al., 2013). ES cells were grown on feeder cells (mitotically inactivated mouse embryonic fibroblasts) in ES cell medium prepared with DMEM (Biochrom) and supplemented with 15\% FCS (PAN), $10 \mathrm{U} / \mathrm{ml}$ Penicillin and Streptomycin, $0.1 \mathrm{mM}$ non-essential amino acids, two mM L-glutamine (Gibco), $0.15 \mathrm{mM} \beta$-mercaptoethanol (Sigma), and $500 \mathrm{U} / \mathrm{ml}$ Lif (Millipore). ES cells were grown at $37^{\circ} \mathrm{C}$ in the presence of $10 \% \mathrm{CO}_{2}$. The medium was changed every two days, and the cells were subcultured or processed at sub-confluence.

\subsection{RNA isolation and gene expression analysis}

Cells were seeded in 6-well plates $\left(10^{5}\right.$ cells per well), incubated to sub-confluence, and collected for RNA isolation. For gene expression analysis, RNA extraction and preparation of cDNA were performed using the methods and reagents described previously (Bedzhov et al., 2013). Primers used in this study and their corresponding UPL probes are presented in Table 1. Relative expression was calculated using the $2^{-\Delta \Delta \mathrm{Ct}}$ method (Livak and Schmittgen, 2001) relative to the wt control and normalized to the housekeeping gene Gapdh. Data represent at least three biological replicates. One-way ANOVA, followed by Tukey's multiple comparison tests, was performed for statistical analysis of group comparisons.
Table 1: Primers and corresponding UPL probes used in qPCR experiments.

\begin{tabular}{|c|c|c|}
\hline $\begin{array}{l}\text { Gene } \\
\text { Name }\end{array}$ & Primer Sequence & UPL probe ID \\
\hline Cdh1 & $\begin{array}{l}\text { F: atcctcgccetgctgatt } \\
\text { R: accaccgttctcctccgta }\end{array}$ & UPL No: 18 \\
\hline$C d h 2$ & $\begin{array}{l}\text { F: tccctgagatacagcgtcact } \\
\text { R: ataatgaagatgcccgttgg }\end{array}$ & UPL No: 17 \\
\hline Eomes & $\begin{array}{l}\text { F: accggcaccaaactgaga } \\
\text { R: aagctcaagaaaggaaacatgc }\end{array}$ & UPL No: 9 \\
\hline Klf4 & $\begin{array}{l}\text { F: cgggaagggagaagacact } \\
\text { R: gagttcctcacgccaacg }\end{array}$ & UPL No: 62 \\
\hline Neurod1 & $\begin{array}{l}\text { F: cgcagaaggcaaggtgtc } \\
\text { R: tttggtcatgtttccacttcc }\end{array}$ & UPL No: 1 \\
\hline Vim & $\begin{array}{l}\text { F: ccaaccttttcttccctgaa } \\
\text { R: ccaacctttcttccctgaa }\end{array}$ & UPL No: 109 \\
\hline Snai1 & $\begin{array}{l}\text { F: gtctgcacgacctgtggaa } \\
\text { R: caggagaatggcttctcacc }\end{array}$ & UPL No: 71 \\
\hline Zeb1 & $\begin{array}{l}\text { F: gccagcagtcatgatgaaaa } \\
\text { R: tatcacaatacgggcaggtg }\end{array}$ & UPL No: 48 \\
\hline Gapdh & $\begin{array}{l}\text { F: agcttgtcatcaacgggaag } \\
\text { R: tttgatgttagtggggtctcg }\end{array}$ & UPL No: 9 \\
\hline
\end{tabular}

\subsection{Microarray analysis}

Microarray analysis for the ki/ki and wt2 cells was described before (Bedzhov et al., 2013). In short, RNA from two independent samples per genotype was hybridized to the GeneChip Mouse Genome 430 v2.0 array (Affymetrix, Inc.). The raw data can be downloaded from the NCBI GEO database (Barrett et al., 2013) (GEO accession number GSE42008). Raw data for the E-cad knockout and the corresponding wild-type cells (wt1) are publicly available and were obtained from the ArrayExpress, accession number E-MEXP-2836 (Soncin et al., 2011). Data analysis was performed in $\mathrm{R} v 4.0 .3$. The data set's quality was examined with the simpleaffy package v2.66.0 (Wilson and Miller, 2005), and expression values and normalization were performed by the GCRMA function, with quantile normalization and excluding features with expression values of $<4$ in all samples. Differential expression analysis was performed with the limma package v 3.46.0 (Ritchie et al., 2015), applying the eBayes function. Heatmaps were plotted using the Heatmap.2 function of the gplots package v3.1.1 (https://cran.r-project.org/web/packages/gplots/index.html ). Using Log2-transformed and normalized expression values, heatmap images were generated, applying Pearson's correlation to calculate hierarchical clustering. Significant genes were calculated by measuring the standard deviation for each row, then subsetting the shortest interval covering half of the values in the data set using the shorth function of the genefilter package v1.72.0 (https://bioconductor.org/packages/release/bioc/html/genef ilter.html), and then calculating the adjusted p-value according to FDR. PCA plots were generated using the stats package's prcomp command and plotted using the autoplot command of the ggplot2 package v3.3.2 (Wickham, 2009). GO enrichment analysis was performed using the GOexpress package v1.24.0 (Rue-Albrecht et al., 2016) with the following parameters: seed $=4543$, permutations $=1000$, and random forest method was selected. 


\section{Results}

In order to understand the impact of E-cad deletion in ES cells, the previously generated mouse models and ES cells where E-cad was replaced for N-cad as a knock-in allele were used (Bedzhov et al., 2013). In the presence of $\mathrm{N}$-cad (either as $\mathrm{ki} / \mathrm{wt}$ or $\mathrm{ki} / \mathrm{ki}$ ), ES cells retained the pluripotency state as opposed to the E-cad ko/ko cells, which lose the pluripotency. The functional loss of E-cad expression in ES cells is not limited to loss of pluripotency; this is why the N-cad knock-in (ki/ki) ES cells present a unique model for studying the consequences of E-cad depletion beyond pluripotency.

\subsection{High correlation between wt and $\mathrm{N}$-cad ki/ki ES cells}

We have previously shown that the $\mathrm{N}$-cad ki/ki cells resembled wt cells morphologically; they grow as compact colonies on feeder cells and express key pluripotencyspecific genes (Bedzhov et al., 2013). This is a continuation of the previous analysis to shed light on the global changes in ki/ki and ko/ko cells. The first analysis revealed that $\mathrm{ki} / \mathrm{ki}$ cells were closely related to wt cells rather than ko/ko cells (Figure 1A). This remarkable similarity is, of course, reflected by the previous findings regarding pluripotency genes and is also reflected by the morphological features mentioned earlier. Moreover, correlation analysis revealed a remarkable $97 \%$ correlation (Figure 1B). As expected, there was no statistically significant correlation between the ko/ko cells compared to either $\mathrm{ki} / \mathrm{ki}$ or wt cells. These results confirmed once more the functional redundancy of E-cad and N-cad in ES cells. The global resemblance of gene expression profiles can also be recognized by looking at a heatmap comparing the three cellular models (Figure 1C). The three-way analysis of samples highlighted the similarities and differences between the three cell lines. However, careful examination of the heatmap also unveils the slight differences between ki/ki and wt ES cells (Figure 1C). This analysis was followed by qPCR experiments to validate the expression profiling results. Accordingly, the status of $C d h 1$ and $C d h 2$ (The official gene symbols for E-cad and N-cad, respectively) expression was confirmed and reflected the genotypic changes in both cell lines (Figure 1D)

A

B

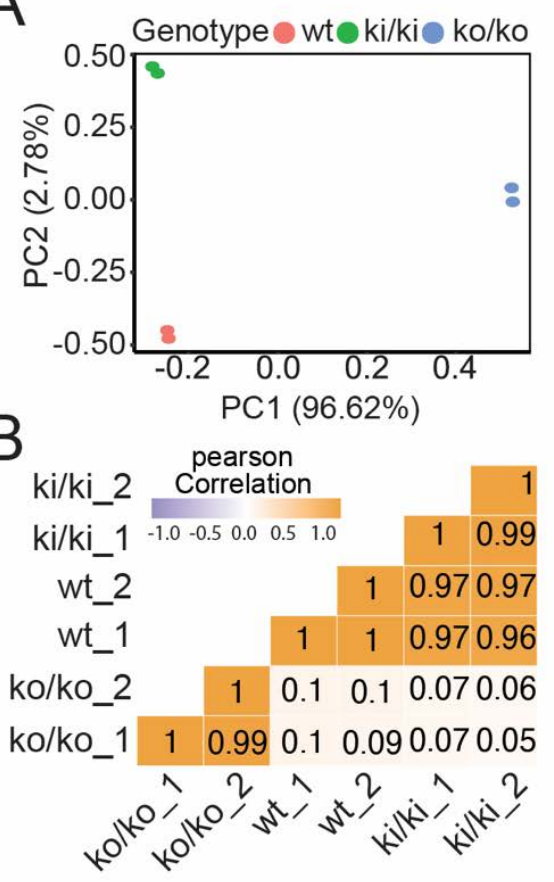

C

D

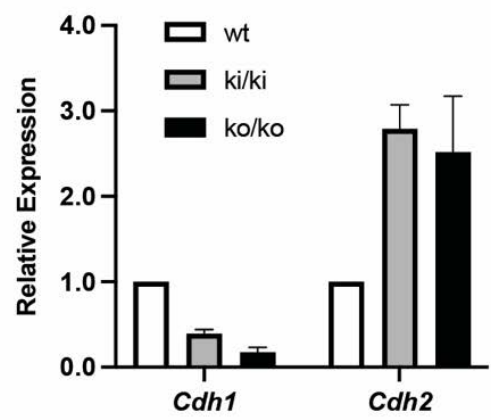

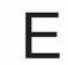

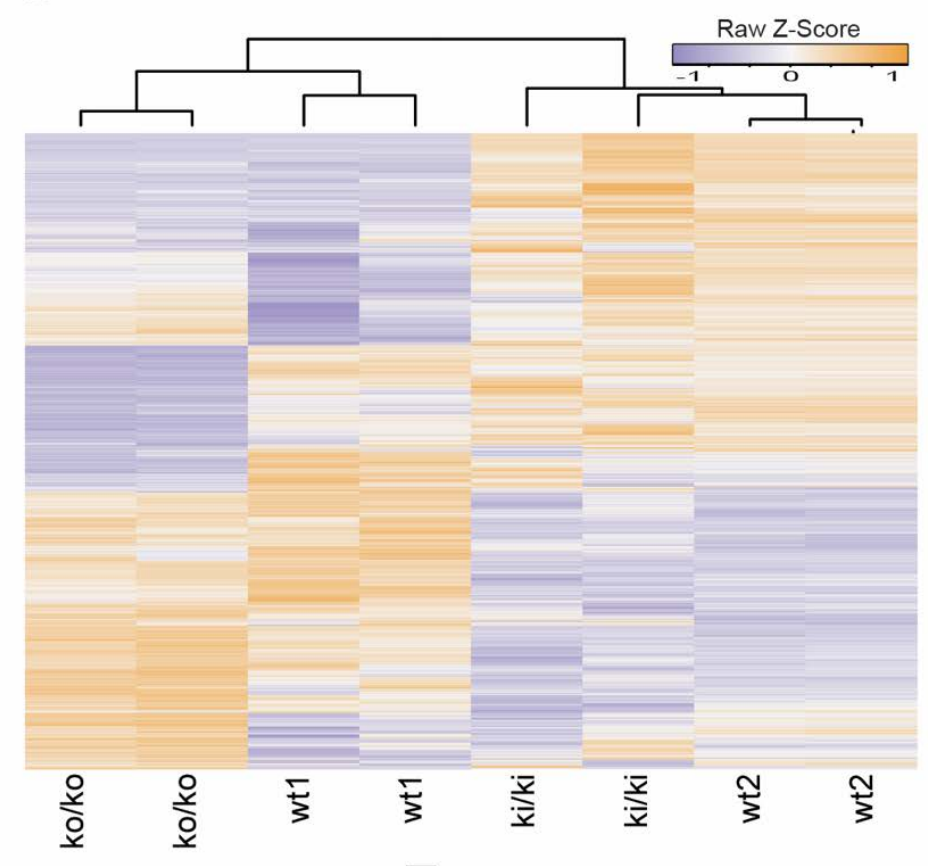

$\mathrm{F}$

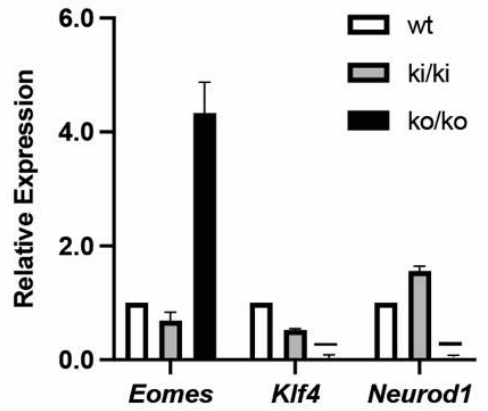

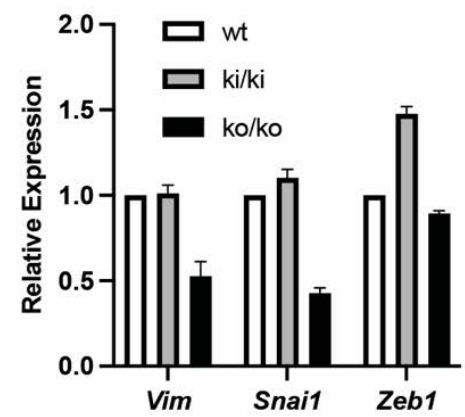

Figure 1. The $\mathbf{N}$-cad ki/ki ES cells are highly similar to the wt ES cells. A) Principal component analysis showing the high similarity between ki/ki and wt cells. PC2 corresponds to less than 3\% difference, while PC1 corresponds to more than 95\% difference. B) Correlation matrix indicating the percentages of similarity between the samples. C) Heatmap drawing of the expression values of all samples used in this study, D-F) qPCR analysis of genes selected to verify the expression data obtained from microarray analysis. Experiments were performed at least 3 times in triplicates. Error bars correspond to the standard error of the mean. Significant values were labeled with the asterisk. 
We have previously studied the expression levels of pluripotency-related genes (Bedzhov et al., 2013). Here, other developmentally relevant genes, such as Eomes, Klf4, and Neurod1 (Figure 1E), as well as genes relevant to the cadherin switching, were selected (Figure 1F). Overall, the expression levels detected using qPCR confirmed the signals obtained from microarray experiments' expression profiles.

\subsection{Common genes differentially expressed in $\mathrm{ki} / \mathrm{ki}$ and ko/ko ES cells}

Studying the differentially expressed genes in ko/ko cells compared to wt ES cells will reveal the impact of Ecad deletion on gene expression, which is reflected by changes in ES physiology. These changes include the known impact on pluripotency. Similarly, examining differentially expressed genes between ki/ki and wt cells will show the impact of the forced $\mathrm{N}$-cad expression on ES cells. Differential expression analyses of ko/ko-wt and ki/ki-wt ES cells were performed using the R-package limma with a cutoff of $\log 2$ 0.6. The number of differentially expressed genes in ko/ko ES cells was 3527 (Figure 2A), and in ki/ki ES cells, it was 1976 (Figure 2B). To better understand E-cad's impact on ES cells, the differentially expressed genes of ko/ko-wt and ki/ki-wt ES cells were compared first. The common genes were categorized into different groups, as illustrated in Figure 2.
A

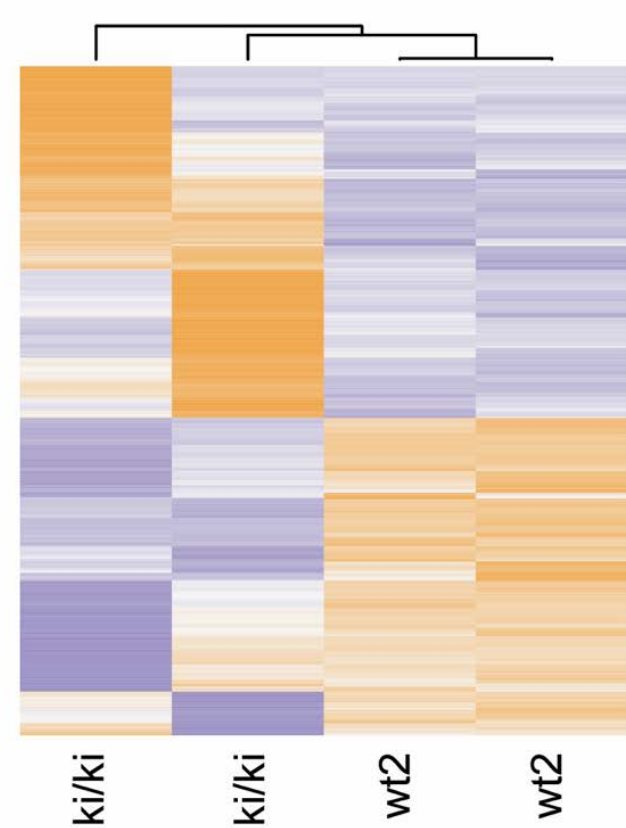

C

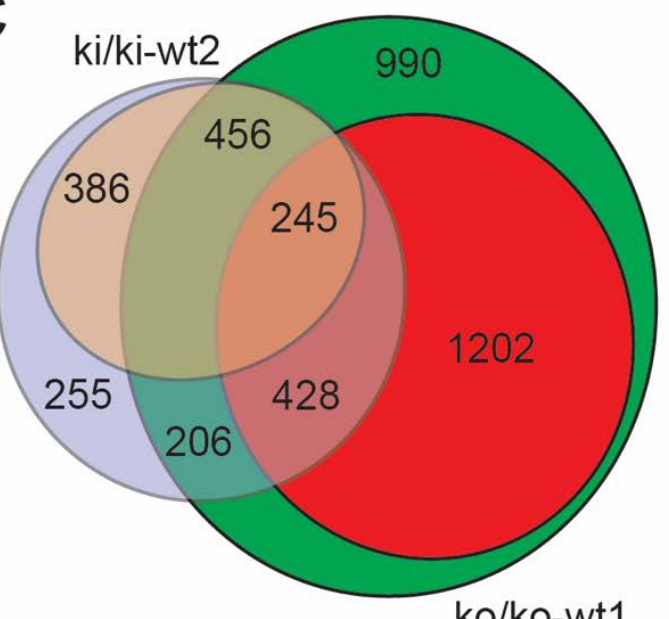

B

Row Z-Score

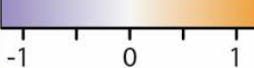

$\mathrm{D}$
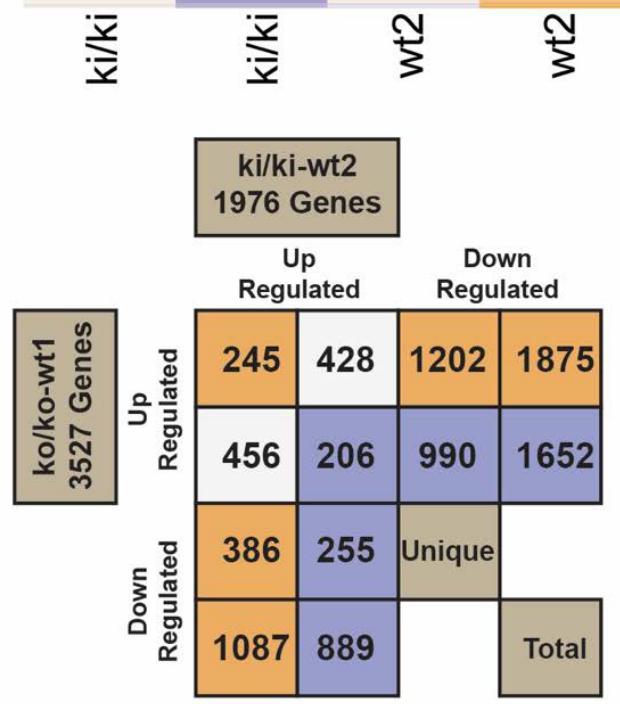

Figure 2. Common differentially expressed genes. A-B) Heatmaps show 1335 differentially expressed genes in ki/ki and ko/ko ES cells. C-D) Numeric representation of commonly expressed genes.

In total, 1335 genes differentially expressed in both $\mathrm{ki} / \mathrm{ki}$-wt and ko/ko-wt comparisons are common. The number of commonly upregulated genes was 245, while 206 genes were found downregulated. The Venn diagram in Figure 2C shows the common and unique areas with the number of genes depicted; the complete data is also summarized as a matrix in Figure 2C. Of note here is the difference in differentially expressed genes in both comparisons. The number of differentially expressed genes in $\mathrm{ko} / \mathrm{ko}$ cells was about two times higher than in $\mathrm{ki} / \mathrm{ki}$ cells, attesting to the similarities between $\mathrm{ki} / \mathrm{ki}$ and wt cells (Figure 2C). As described in materials and methods, 
common genes differentially expressed were subjected to sequential filtering to find significant genes. The number of remaining genes that remained after applying the adjusted p-value of 0.01 filter was 280 (Supplementary Table S1). These genes were also plotted in the heatmap in supplementary Figure S1 and revealed the remarkable resemblance of $\mathrm{ki} / \mathrm{ki}$ ES cells to their wt counterparts. The heatmap view also revealed the subtle differences between

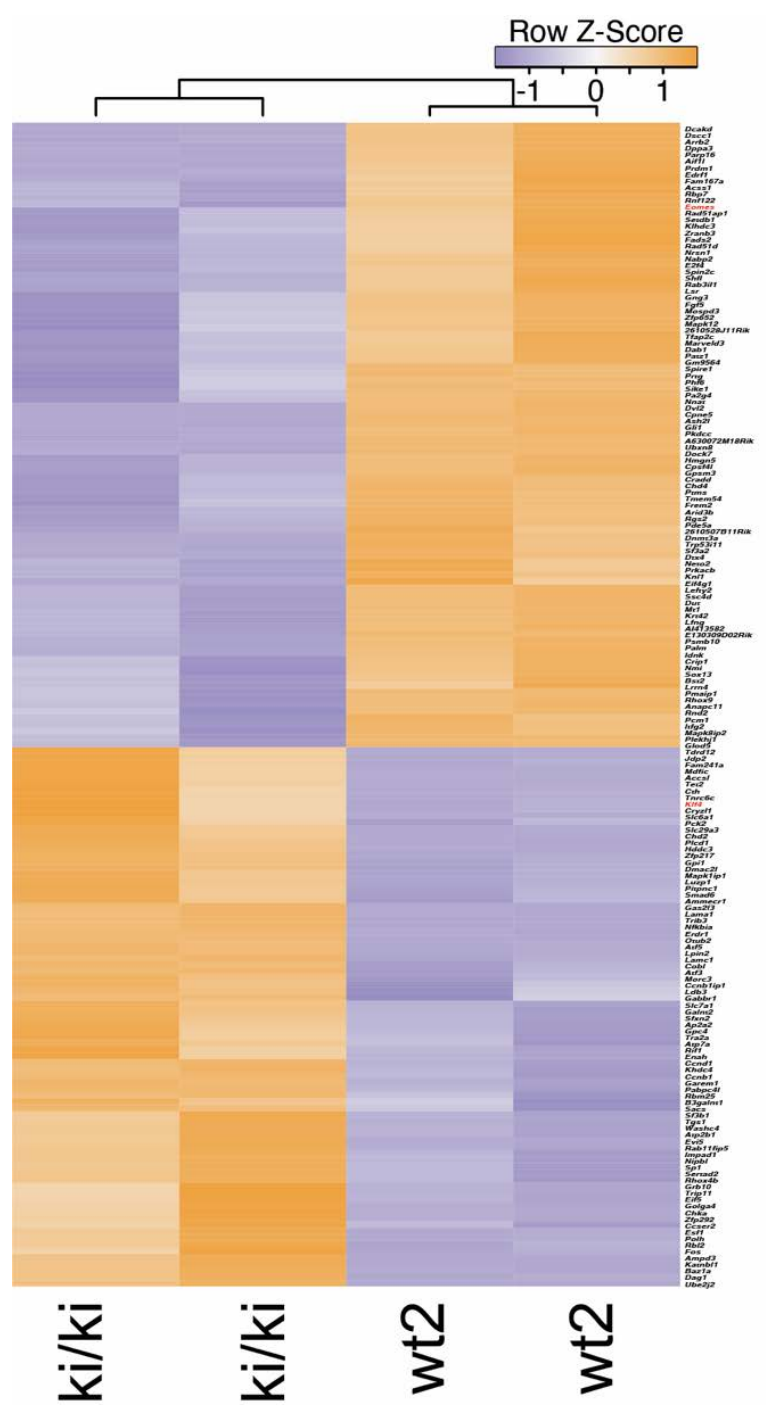

ki/ki ES cells and wt cells. To better understand these differences, subsetting the common differentially expressed genes for the $\mathrm{ki} / \mathrm{ki}$ and wt samples from the dataset was performed and followed by applying the filtering as done in the previous analysis. As a result, 179 differentially expressed genes in the ki/ki ES cells with an adjusted p-value of $<0.05$ were found (Figure $3 \mathrm{~A}$ and Supplementary Table S2).

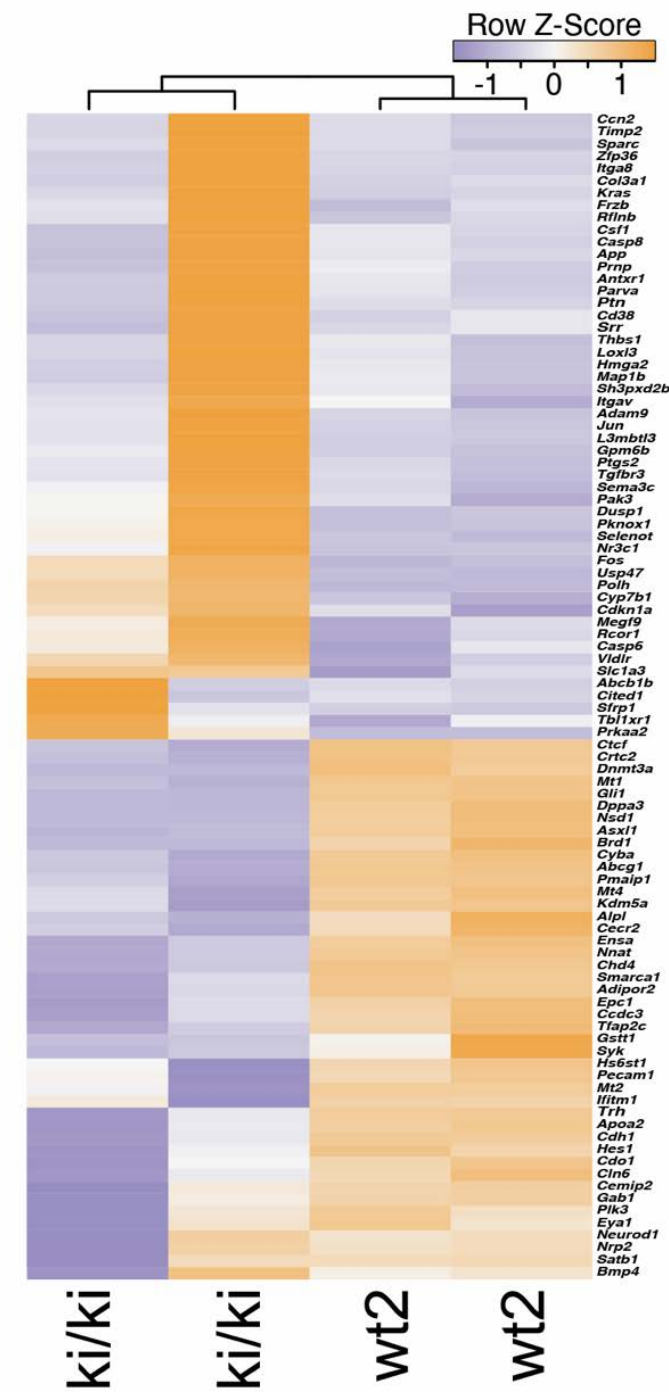

Figure 3. Commonly upregulated and downregulated genes in the ki/ki ES cells. A) Heatmap of the common differentially expressed genes in ki/ki cells. One hundred seventy-nine genes with an adjusted p-value of 0.05 or less were plotted here. Upregulated or downregulated genes in both genotypes were subjected to GO enrichment analysis. The heatmap in B represents 95 genes that were found as enriched.

\subsection{GO enrichment analysis of common genes differentially expressed in $\mathrm{ki} / \mathrm{ki}$ and ko/ko ES cells}

To determine the relevance of the differentially expressed genes in ki/ki cells, commonly expressed upand downregulated genes were included in the GO enrichment analysis. A total of 451 genes (245 upregulated and 206 downregulated) were subjected to GO enrichment analysis using the R-package GOexpress. This analysis revealed significant enrichment in 95 genes belonging to 19 different biological processes. The expression pattern of these genes is presented in the heatmap in Figure 3B. The GO enrichment analysis revealed significant changes in genes within the response to the drug biological process (20 genes: $\sim 14 \%$ ).
Moreover, chromatin organization (18 genes: 12\%) related genes and genes related to angiogenesis (14 genes: $\sim 10 \%$ ) were also significantly represented. A summary of the GO enrichment analysis results is depicted in the pie chart in figure 4A. Eighteen genes belonging to the chromatin organization biological process were mainly downregulated in response to Cdh1 depletion in the ki/ki cells (Figure 4B), suggesting a positive correlation between these biological processes and E-cad function. On the other hand, genes related to EMT were found to be upregulated in the $\mathrm{ki} / \mathrm{ki}$ cells, suggesting a tendency to shift to a mesenchymal state in the absence of E-cad (Figure 4C). 
A

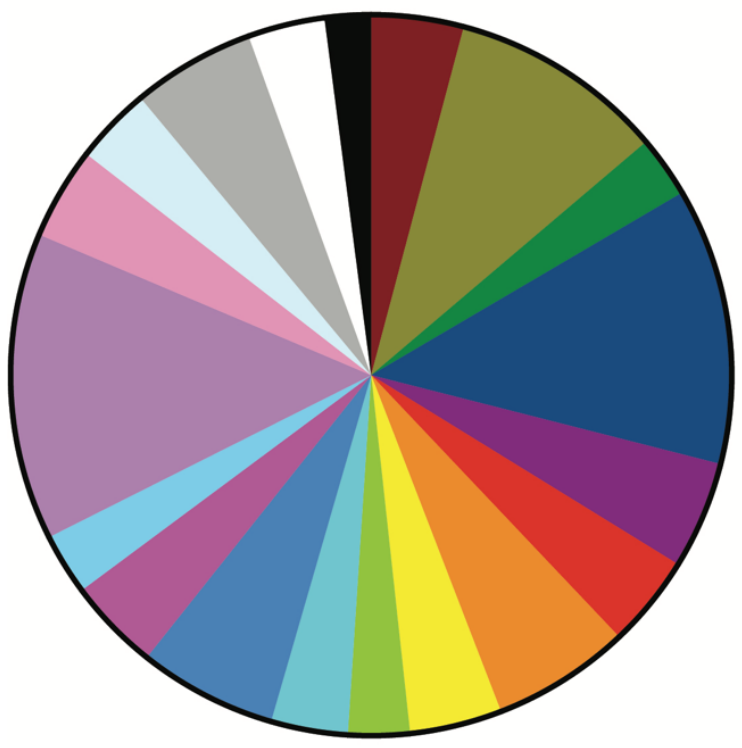

$4.14 \%$ ossification

$9.66 \%$ angiogenesis

$2.76 \%$ epithelial to mesenchymal transition

$12.41 \%$ chromatin organization

$4.83 \%$ cholesterol metabolic process

$4.14 \%$ response to radiation

$6.21 \%$ response to glucose

$4.14 \%$ positive regulation of cell death

$2.76 \%$ neural tube development

$3.45 \%$ pituitary gland development

$6.21 \%$ response to cytokine

$4.14 \%$ substrate adhesion-dependent cell spreading

$2.76 \%$ somatic stem cell population maintenance

$13.79 \%$ response to drug

$4.14 \%$ positive regulation of neuron apoptotic process

$3.45 \%$ positive regulation of fat cell differentiation

$5.52 \%$ response to glucocorticoid

$3.45 \%$ cellular response to cadmium ion

$2.07 \%$ toxin transport
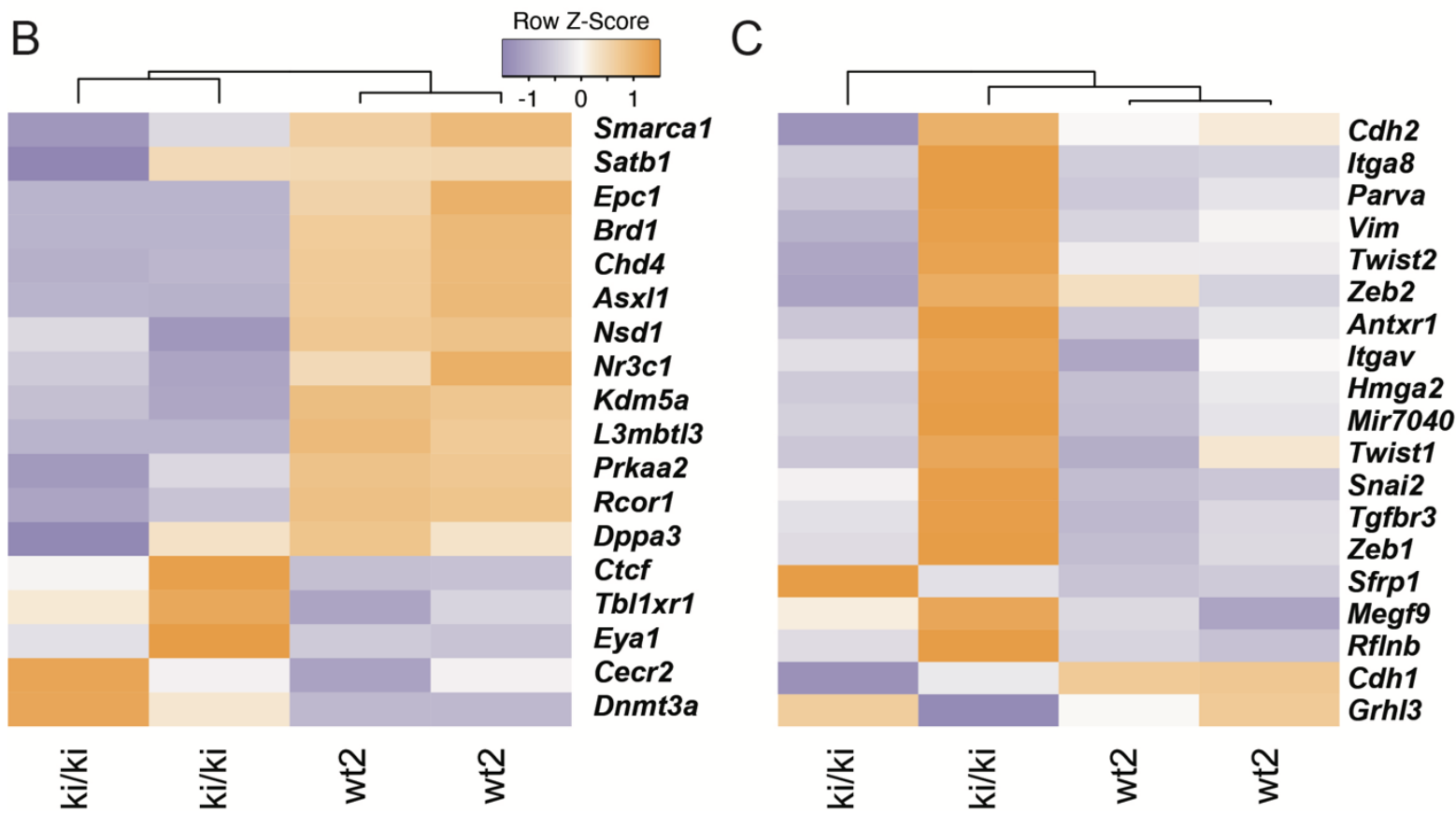

Figure 4. GO enrichment analysis identifies significant biological processes affected by E-cad depletion. A) Nineteen biological process GO terms were enriched in ki/ki ES cells, and their percentage is represented in the pie chart. B) Chromatin organization genes were mostly downregulated in ki/ki ES cells. C) EMT-related genes were upregulated ki/ki ES 1 cells.

Other gene groups are also presented in Supplemental Figure 2. Of note, genes belonging to three developmental processes were mapped in supplementary Figure S2; the ossification gene group was mainly upregulated in the absence of E-cad. In contrast, genes belonging to the pituitary gland development were mainly downregulated in the ki/ki cells. The neural tube development genes were mixed (Figure S2A). Genes in the angiogenesis (Figure $\mathrm{S} 2 \mathrm{~B}$ ), apoptosis (Figure S2C), and response to drugs (Figure S2D) were mainly upregulated as a result of E-cad absence. A detailed description of all biological processes represented by the 95 enriched genes is displayed in Table 2. 
Table 2: Detailed descriptions of the identified GO enrichment analysis showing significant biological process terms and corresponding genes.

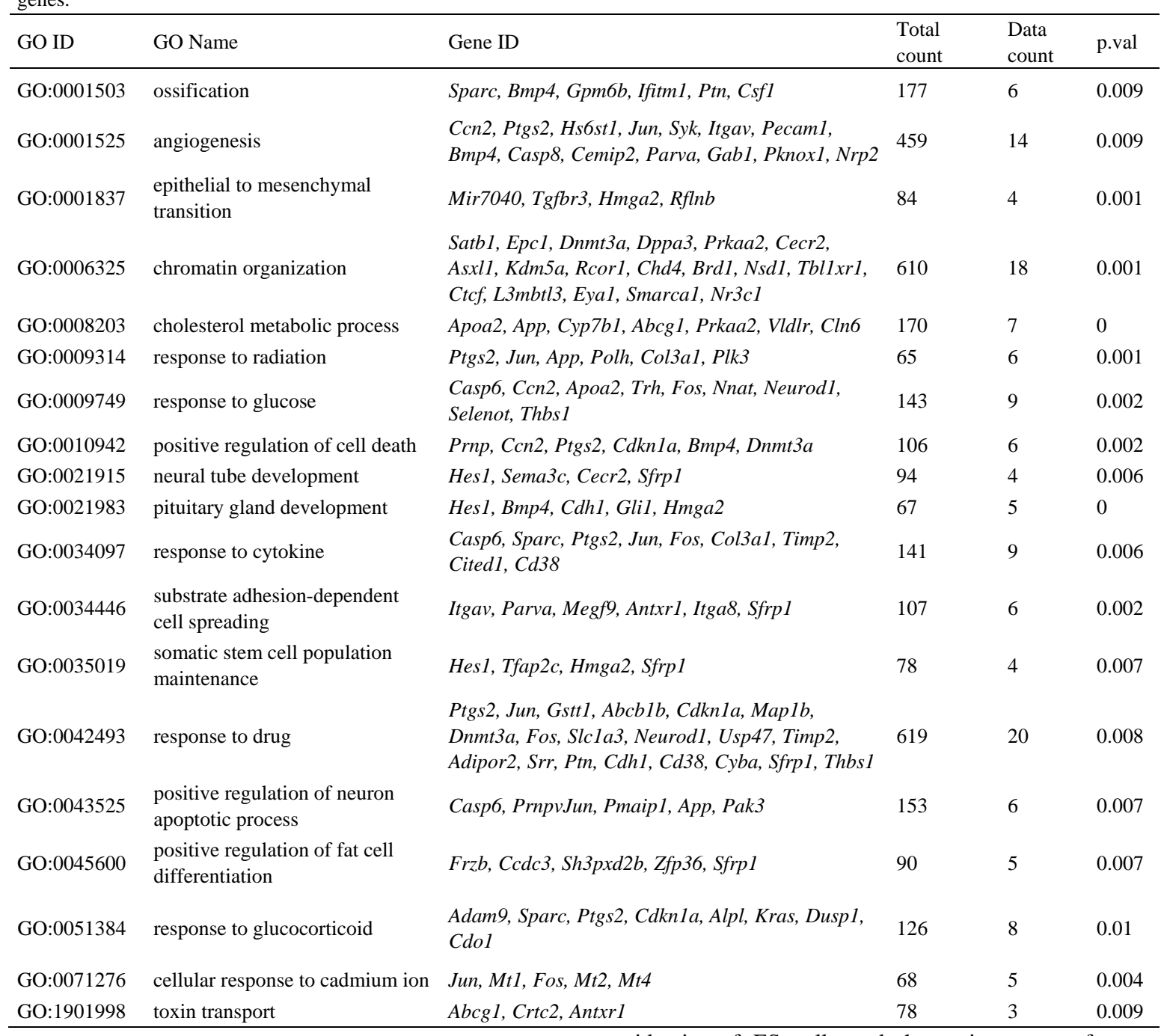

\section{Discussion}

This manuscript addresses the impact of E-cad deletion in ES cells while retaining the pluripotency state, taking advantage of our previously published cellular model in which the expression of $\mathrm{N}$-cad as a knock-in allele in the E-cad locus was performed. The expression profiles of ES cells with deleted E-cad (the ko/ko cells) or N-cad as a knock-in allele in the E-cad locus (the ki/ki cells) were compared to the wild type ES cells using an in silico approach. The analysis revealed a slight shift to the mesenchymal gene signature by upregulating EMT-related genes. On the other hand, a decrease in the expression of chromatin organization-related genes was observed in the absence of E-cad. The gene replacement model analyzed here proved to be a powerful tool to study aspects of E-cad biology. The first studies of replacing E-cad with $\mathrm{N}$-cad revealed that $\mathrm{N}$-cad could not replace $\mathrm{E}$-cad function during the formation of the trophectoderm (Kan et al., 2007); this was illustrated by the specific crosstalk between E-cad and Igf1r signaling providing a survival signal that cannot be reproduced when $\mathrm{N}$-cad is expressed instead of E-cad (Bedzhov et al., 2012). Several reports described the essential role E-cad plays in defining the identity of ES cells and the maintenance of stemness (Chen et al., 2010, Soncin et al., 2011).

Furthermore, E-cad was also described as an essential facilitator of the reprogramming of iPSCs; it could even replace members of the Yamanaka cocktail used in iPSC derivation (Li et al., 2010, Redmer et al., 2011). The high homology between E-cad and $\mathrm{N}$-cad results in a significant redundancy in ES cells' pluripotency. As opposed to trophectoderm development, ki/ki ES cells injected in blastocysts can develop up to embryonic day E8.5, showing that $\mathrm{N}$-cad could replace E-cad in these chimeric embryos (Bedzhov et al., 2013). Notably, the N-cad ki/ki ES cells maintained pluripotency, and the resemblance of the gene expression profile to the wild-type ES cells was distinctive. Despite the global comparability of gene expression profiles of $\mathrm{N}$-cad ki/ki ES cells and the wildtype ES cells, subtle differences were observed. These differences are evident when looking at subsetted heatmaps encompassing genes within the biological processes enriched using the GO enrichment analysis.

The results presented here suggest that the loss of Ecad in ES cells results in the foundation of a partial EMTlike state; while cells retain adhesive characteristics, due to $\mathrm{N}$-cad expression, they upregulate specific EMT associated genes such as Zeb1 and Tgfbr3 and downregulate essential epithelial genes such as Grhl3. 
Loss of Grhl3 was previously shown to result in a robust mesenchymal phenotype during MET initiation (Alotaibi et al., 2015). The effects of E-cad depletion have been studied in the non-tumorigenic MCF10A cells. The cells presented significant changes associated with tissue remodeling and cell-substrate attachment proteins such as ITGA1. On the other hand, they did not show a similar increase in EMT-related genes (Chen et al., 2014). The observed increase in Itga8 and Itgav gene expression is in agreement with their findings. E-cad deletion also affected several developmental genes, particularly the ossification genes, and some of the neural tube development-related genes were mainly upregulated. This was associated with changes in differentiation genes and suggested a link between the changes in the EMT-associated genes and the development-related or differentiation-related genes. Cellular reprogramming is known to include EMT and MET (Li et al., 2010), and the differentiation state of cells is also expressed in terms of epithelial-like or mesenchymal-like. A recent report showed that a sequential EMT/MET is required to differentiate hESCs to the hepatic lineage. This process involves an autocrine TGF $\beta$ signaling loop resulting in an upregulation of EMT inducers and downregulation of E-cad expression ( $\mathrm{Li}$ et al., 2017). Concerning reprogramming, the observed downregulation in the chromatin organization gene signature was profound, except for Ctcf and Dnmt3a, which regulate chromatin architecture and DNA methylation. Epigenetic reprogramming is closely associated with fate determination, differentiation, and cellular reprogramming. Previous studies indicated a critical role for E-cad in reprogramming MEFs into iPSCs (Li et al., 2010, Redmer et al., 2011).

Furthermore, genetic ablation of E-cad prevented iPSC reprogramming (Redmer et al., 2011, Bedzhov et al., 2013). This reprogramming is associated with global epigenetic reorganization, resetting the chromatin infrastructure from a differentiated somatic state to an undifferentiated pluripotent state (Hochedlinger and Jaenisch, 2015). E-cad's importance to ES cells in terms of pluripotency is well known, and its essential role in the reprogramming to the pluripotent state is central to the establishment of MET that precedes the pluripotency network activation. In the absence of E-cad, the tendency to shift to a more differentiated state as illustrated by changes in chromatin organization genes and EMTassociated genes shed light onto new aspects of E-cad functions in ES cells. It will be of great interest to experimentally corroborate these novel findings, which will significantly benefit the field of stem cell biology and eventually personalized medicine. Stem cell therapy and personalized medicine are considered the future of medicine (Strauer and Kornowski, 2003). The scope of personalized medicine goes beyond common diseases such as cancer and is considered for developmental disorders or rare diseases as well (Garcia-Castro and Singec, 2017). Hematopoietic stem cells are used to treat leukemia (Tian et al., 2016), and recent examples can also be seen in studies related to Parkinson's disease (Parmar et al., 2020). With this in mind, it is imperative to acknowledge the importance of understanding changes in molecular pathways and gene signatures when planning novel therapeutics.

\section{Acknowledgments}

We would like to thank Dr. Gökhan Karakülah and Prof. Uygar Tazebay for carefully reading the manuscript. This study was financed by İzmir Biomedicine and Genome Center institutional funds. The author discloses no conflict of interest that may have influenced either the conduct or the presentation of the research.

\section{Supplementary Data}

Supplementary Data is available online at the Mendeley data website (http://dx.doi.org/10.17632/wd66b4gb4d.2).

\section{References}

Alotaibi H, Basilicata MF, Shehwana H, Kosowan T, Schreck I, Braeutigam C, Konu O, Brabletz T and Stemmler MP. 2015. Enhancer cooperativity as a novel mechanism underlying the transcriptional regulation of E-cadherin during mesenchymal to epithelial transition. Biochimica Et Biophysica Acta-Gene Regulatory Mechanisms, 1849(6): 731-742.

Barrett T, Wilhite SE, Ledoux P, Evangelista C, Kim IF, Tomashevsky M, Marshall KA, Phillippy KH, Sherman PM, Holko M, Yefanov A, Lee H, Zhang N, Robertson CL, Serova N, Davis S and Soboleva A. 2013. NCBI GEO: archive for functional genomics data sets--update. Nucleic Acids Res, 41(Database issue): D991-995.

Bedzhov I, Alotaibi H, Basilicata MF, Ahlborn K, Liszewska E, Brabletz T and Stemmler MP. 2013. Adhesion, but not a specific cadherin code, is indispensable for ES cell and induced pluripotency. Stem Cell Res, 11(3): 1250-1263.

Bedzhov I, Liszewska E, Kanzler B and Stemmler MP. 2012. Igf1r signaling is indispensable for preimplantation development and is activated via a novel function of E-cadherin. PLoS Genet, 8(3): e1002609.

Boyer LA, Plath K, Zeitlinger J, Brambrink T, Medeiros LA, Lee TI, Levine SS, Wernig M, Tajonar A, Ray MK, Bell GW, Otte AP, Vidal M, Gifford DK, Young RA and Jaenisch R. 2006. Polycomb complexes repress developmental regulators in murine embryonic stem cells. Nature, 441(7091): 349-353.

Chen A, Beetham H, Black MA, Priya R, Telford BJ, Guest J, Wiggins GA, Godwin TD, Yap AS and Guilford PJ. 2014. Ecadherin loss alters cytoskeletal organization and adhesion in nonmalignant breast cells but is insufficient to induce an epithelialmesenchymal transition. BMC Cancer, 14: 552.

Chen T, Yuan D, Wei B, Jiang J, Kang J, Ling K, Gu Y, Li J, Xiao L and Pei G. 2010. E-cadherin-mediated cell-cell contact is critical for induced pluripotent stem cell generation. Stem Cells, 28(8): 1315-1325.

Garcia-Castro J and Singec I. 2017. Prospects of Pluripotent and Adult Stem Cells for Rare Diseases. Adv Exp Med Biol, 1031: 371-386.

Hochedlinger K and Jaenisch R. 2015. Induced Pluripotency and Epigenetic Reprogramming. Cold Spring Harb Perspect Biol, 7(12).

Kan NG, Stemmler MP, Junghans D, Kanzler B, de Vries WN, Dominis M and Kemler R. 2007. Gene replacement reveals a specific role for E-cadherin in the formation of a functional trophectoderm. Development, 134(1): 31-41.

Larue L, Antos C, Butz S, Huber O, Delmas V, Dominis M and Kemler R. 1996. A role for cadherins in tissue formation. Development, 122(10): 3185-3194. 
Li Q, Hutchins AP, Chen Y, Li S, Shan Y, Liao B, Zheng D, Shi X, Li Y, Chan WY, Pan G, Wei S, Shu X and Pei D. 2017. A sequential EMT-MET mechanism drives the differentiation of human embryonic stem cells towards hepatocytes. Nat Commun, 8: 15166 .

Li R, Liang J, Ni S, Zhou T, Qing X, Li H, He W, Chen J, Li F, Zhuang Q, Qin B, Xu J, Li W, Yang J, Gan Y, Qin D, Feng S, Song H, Yang D, Zhang B, Zeng L, Lai L, Esteban MA and Pei D. 2010. A mesenchymal-to-epithelial transition initiates and is required for the nuclear reprogramming of mouse fibroblasts. Cell Stem Cell, 7(1): 51-63.

Livak KJ and Schmittgen TD. 2001. Analysis of relative gene expression data using real-time quantitative PCR and the 2(-Delta Delta C(T)) Method. Methods, 25(4): 402-408.

Maherali N, Sridharan R, Xie W, Utikal J, Eminli S, Arnold K, Stadtfeld M, Yachechko R, Tchieu J, Jaenisch R, Plath K and Hochedlinger K. 2007. Directly reprogrammed fibroblasts show global epigenetic remodeling and widespread tissue contribution. Cell Stem Cell, 1(1): 55-70.

Mikkelsen TS, Ku M, Jaffe DB, Issac B, Lieberman E, Giannoukos G, Alvarez P, Brockman W, Kim TK, Koche RP, Lee W, Mendenhall E, O'Donovan A, Presser A, Russ C, Xie X, Meissner A, Wernig M, Jaenisch R, Nusbaum C, Lander ES and Bernstein BE. 2007. Genome-wide maps of chromatin state in pluripotent and lineage-committed cells. Nature, 448(7153): 553560 .

Niwa H. 2007. How is pluripotency determined and maintained? Development, 134(4): 635-646.

Parmar M, Grealish S and Henchcliffe C. 2020. The future of stem cell therapies for Parkinson disease. Nat Rev Neurosci, 21(2): 103-115.

Redmer T, Diecke S, Grigoryan T, Quiroga-Negreira A, Birchmeier W and Besser D. 2011. E-cadherin is crucial for embryonic stem cell pluripotency and can replace OCT4 during somatic cell reprogramming. EMBO Rep, 12(7): 720-726.

Ritchie ME, Phipson B, Wu D, Hu Y, Law CW, Shi W and Smyth GK. 2015. limma powers differential expression analyses for RNA-sequencing and microarray studies. Nucleic Acids Res, 43(7): e47.
Rue-Albrecht K, McGettigan PA, Hernandez B, Nalpas NC, Magee DA, Parnell AC, Gordon SV and MacHugh DE. 2016. GOexpress: an R/Bioconductor package for the identification and visualisation of robust gene ontology signatures through supervised learning of gene expression data. BMC Bioinformatics, 17: 126.

Silva J, Nichols J, Theunissen TW, Guo G, van Oosten AL, Barrandon O, Wray J, Yamanaka S, Chambers I and Smith A. 2009. Nanog is the gateway to the pluripotent ground state. Cell, 138(4): $722-737$.

Soncin F, Mohamet L, Eckardt D, Ritson S, Eastham AM, Bobola N, Russell A, Davies S, Kemler R, Merry CL and Ward CM. 2009. Abrogation of E-cadherin-mediated cell-cell contact in mouse embryonic stem cells results in reversible LIF-independent self-renewal. Stem Cells, 27(9): 2069-2080.

Soncin F, Mohamet L, Ritson S, Hawkins K, Bobola N, Zeef L, Merry CL and Ward CM. 2011. E-cadherin acts as a regulator of transcripts associated with a wide range of cellular processes in mouse embryonic stem cells. PLoS One, 6(7): e21463.

Strauer BE and Kornowski R. 2003. Stem cell therapy in perspective. Circulation, 107(7): 929-934.

Takahashi K and Yamanaka S. 2006. Induction of pluripotent stem cells from mouse embryonic and adult fibroblast cultures by defined factors. Cell, 126(4): 663-676.

Tian H, Qu Q, Liu L and Wu D. 2016. Advances in Stem Cell Therapy for Leukemia. Curr Stem Cell Res Ther, 11(2): 158-165.

Wheelock MJ, Shintani Y, Maeda M, Fukumoto Y and Johnson KR. 2008. Cadherin switching. J Cell Sci, 121(Pt 6): 727-735.

Wickham H (2009). ggplot2 City.

Wilson CL and Miller CJ. 2005. Simpleaffy: a BioConductor package for Affymetrix Quality Control and data analysis. Bioinformatics, 21(18): 3683-3685.

Yamanaka S. 2020. Pluripotent Stem Cell-Based Cell TherapyPromise and Challenges. Cell Stem Cell, 27(4): 523-531.

Ying QL, Wray J, Nichols J, Batlle-Morera L, Doble B, Woodgett J, Cohen P and Smith A. 2008. The ground state of embryonic stem cell self-renewal. Nature, 453(7194): 519-523. 